\title{
The National Bowel Cancer Screening Program: time to achieve its potential to save lives
}

\author{
Hooi C Eea,e and James St John b,c,d \\ a Department of Gastroenterology, Sir Charles Gairdner Hospital, Perth, WA, Australia \\ b Cancer Prevention Division, Cancer Council Victoria, Melbourne, Australia \\ c Department of Medicine, Royal Melbourne Hospital, University of Melbourne, VIC, Australia \\ ' Department of Gastroenterology, Royal Melbourne Hospital, VIC, Australia \\ e Corresponding author: hooi.ee@health.wa.gov.au
}

\section{Article history}

Publication date: July 2019

Citation: Ee HC, St John J. The National Bowel Cancer Screening Program: time to achieve its potential to save lives. Public Health Res Pract. 2019;29(2):e2921915. https://doi.org/10.17061/phrp2921915

\section{Key points}

- The National Bowel Cancer Screening Program began in 2006 and will be fully rolled out by 2020 , with biennial immunochemical faecal occult blood testing offered to people aged 5074 years

- Data show that participants have earlier cancer diagnoses and a 15\% mortality reduction compared with non-participants

- Increasing participation will improve the program's effectiveness and costeffectiveness, with clinical, social and economic benefits all increasing in step with participation rates

- The program is under constant review to ensure maximum benefits and minimum harms

\section{Abstract}

Objectives: Bowel cancer is the second most common cause of cancer deaths in Australia, affecting both men and women. The National Bowel Cancer Screening Program (NBCSP) began in 2006 with the aim of reducing the morbidity and mortality from bowel cancer in Australia. It is based on level I evidence of mortality reduction with screening using the faecal occult blood test (FOBT).

Type of program or service: The NBCSP is a world-first national program using the immunochemical FOBT (iFOBT), beginning as a staged rollout limited to people aged 55 and 65 years. By 2020, rollout will be complete, with biennial screening for people aged between 50 and 74 years. The program is managed by the Australian Government Department of Health, in partnership with the states and territories.

Methods: Mailing of iFOBT kits is organised through a national register and participants with a positive test are recommended for colonoscopy by the usual care pathway. Outcomes are reported to a national register, with the Australian Institute of Health and Welfare providing crucial support with data monitoring and analyses. This paper analyses the program's implementation and outcomes to date, and looks at whether it is on track to reach its full potential

Results: Program participants have been shown to have earlier cancer diagnoses and a 15\% mortality reduction after accounting for lead-time bias. Modelling analyses predict that increasing participation from the current $41 \%$ will result in further reductions in mortality and improvements in cost-effectiveness, which should eventually deliver net savings to the Australian Government.

Lessons learnt: The initiation and success of the NBCSP has been founded on strong evidence, but there remain areas for improvement, including capture of outcomes data and timely colonoscopy access. On current evidence, increasing participation among the established 50-74-year-old cohort will yield the strongest investment in improved outcomes. Future technological change could present opportunities in risk-based, personalised prevention and screening. Expanding the age range, for example, by starting screening at 45 years, is currently under review. Importantly, maintenance and development of the program should be driven by strong evidence to ensure its ongoing success as a major Australian health initiative. 


\section{Background}

Bowel (or colorectal) cancer is a major population health problem in Australia with projections of 16398 cases and 5597 deaths in 2019, making it the nation's second most common cause of cancer deaths. ${ }^{1}$ Its incidence increases markedly after 40 years of age, with diet, increasing body weight and reduced physical activity being dominant modifiable risk factors. ${ }^{2}$

Bowel cancer arises from benign adenomatous polyps; removal of these polyps will prevent cancer development. Diagnosing bowel cancer at earlier stages improves prognosis. Assessing for invisible (occult) blood in the stool was long conceived as a simple, non-invasive means of detecting cancer (and advanced adenomatous polyps). With bowel cancer being an exemplary disease for population screening, three major randomised controlled trials using the faecal occult blood test (FOBT) were performed in the 1990s, demonstrating a 15-30\% reduction in mortality. ${ }^{3}$

Such compelling evidence led to an Australian Health Technology Advisory Committee report in 1997 recommending that a bowel cancer screening program be started for Australians from age 50 onwards, but that it should first be assessed with a pilot program. ${ }^{3}$ A 1998 meta-analysis including these three trials concluded there was level 1 evidence (i.e. evidence from at least one randomised controlled trial) that population screening with the FOBT reduced mortality from bowel cancer. ${ }^{4}$ In 1999, the National Health and Medical Research Council endorsed inaugural clinical practice guidelines recommending population bowel cancer screening with FOBT for 50-74-year-olds. ${ }^{5}$

However, there were concerns about potential negative effects of a bowel cancer screening program, including concerns about imposing the test, reluctance to participate, difficulties with understanding test performance and anxieties caused by a positive test. As a result, the Australian Bowel Cancer Screening Pilot Program was designed to assess these and other factors. The pilot ran from 2002 to 2004, in one rural and two urban locations, concluding that a national program would be feasible and acceptable in the Australian setting. ${ }^{6}$ One important advance in the pilot was to use the more sensitive and specific immunochemical FOBT (iFOBT) instead of the conventional guaiac chemical test (gFOBT).

\section{The National Bowel Cancer Screening Program (NBCSP)}

The National Bowel Cancer Screening Program (NBCSP) finally started in 2006, managed by the Australian Government Department of Health in partnership with state and territory governments. It was the first national program in the world to use the iFOBT kit. However, it was restricted to people in only two age bands, 55 and
65 years. Although this was partly to ensure colonoscopy and treatment services were able to cope with the increased demand, the initial restriction to two age groups and slowness to develop an implementation plan were also thought to be driven by expenditure concerns at the national level.

Kits were mailed out as determined by a central register. Participants returned their completed kits by mail to a specific pathology provider. The central register informed the participant and their general practitioner (GP) of the results. Participants found to be iFOBTpositive were advised to visit their GP for discussion and, if appropriate, referral for colonoscopy through the usual care pathway. GPs had a critical role in guiding patient decisions on participating, deferring or opting out of the program.

Subsequent rollout was slow; in 2008, 50-year-olds were also included, but without further commitment for expansion. Early signs of benefit dramatically demonstrated that $40 \%$ of NBCSP-detected cancers were stage I and $3 \%$ were stage IV, in contrast to symptomatically presenting cancers where the ratios were $14 \%$ and $15 \%$, respectively. ${ }^{7}$

However, there remained discontent at screening only those aged 50, 55 and 65 years, when screening older ages with a higher cancer prevalence was shown to be more cost-effective. ${ }^{8}$ Subsequent modelling showed that full program implementation - biennial iFOBT screening for 50-74-year-olds - would reduce mortality by 15-25\% at a cost-effective \$25 000-\$41667 per life year gained. ${ }^{9}$ In 2013, a population-based analysis in South Australia showed that $39 \%$ of cancers among NBCSP participants were diagnosed at stage I and 3\% at stage IV, in contrast to $19 \%$ stage I and $12 \%$ stage IV among non-participants - similar to the 2009 report. ${ }^{10}$ Further modelling showed that completion of rollout by 2020 would save $100 \%$ more lives (34 869) over the next 40 years compared to a static program. ${ }^{11}$

The program was gradually expanded from 2013 onwards to cover ages up to 74 years. Finally, a quarter century after the production of level I evidence for mortality reduction, the last two age groups (52 and 56 years) were included in the invitation list in January 2019, so that biennial screening will cover the entire target population by December 2020 .

\section{Screening outcomes}

In 2014, the Australian Institute of Health and Welfare (AIHW) reported outcomes for NBCSP invitees compared with those not invited in the 2006-2008 period. Invitees showed a bowel cancer mortality advantage of $15 \%$ after correcting for lead-time bias. When compared with participants, non-participants had 38\% higher odds of being diagnosed at a more advanced stage of cancer. ${ }^{12}$ The iFOBT positive predictive value for cancer was 3.6\%, with a test sensitivity of $83 \%$ and specificity of $93 \%$. 
Of the 297378 participants with negative screening results, $176(0.06 \%)$ had a false negative result, with cancer diagnosed over the following 2-year period. With the positive predictive value of $3.6 \%$, the likelihood of harbouring bowel cancer is 60 times greater in those who are iFOBT-positive than negative ${ }^{13}$, an important point when considering efficient use of colonoscopy. More recent analysis (2018) of outcomes of participants from 2006 to 2010 showed similar findings to the earlier report. ${ }^{14}$ The NBCSP therefore appears set to fulfil its primary objective of reducing the incidence, morbidity and mortality of bowel cancer.

Between 2015 and 2016, more than 3 million kits were mailed out. The overall participation rate was $41 \%$, although substantially lower rates were found among Indigenous Australians, many groups where a language other than English was dominant at home, people with disabilities, younger age groups and people from very remote locations. ${ }^{2}$ The iFOBT positivity rate was $8.1 \%$ and, of these, $68 \%$ were recorded as having undergone follow-up colonoscopy. The median waiting time for colonoscopy was 54 days, with longer waiting times for the very remote and lower socio-economic groups. Data from colonoscopy pathology are less accurate due to problems with data collection, but approximately $4 \%$ of colonoscopies detected cancer and $57 \%$ found no significant abnormality. ${ }^{2}$ While over half of the participants with a iFOBT positive result do not have cancer or adenoma, the screening test result may lead to anxiety, as well as participants undergoing colonoscopy which carries small risks. For instance, in 2016, 6 cases per 10,000 colonoscopies required a hospital admission within 30 days of the procedure for a possible complication. $^{2}$

\section{Participation in screening}

With full rollout and a participation rate of $40 \%$, the NBCSP is expected to save 59000 bowel cancer deaths over the next 25 years, at a cost saving of

$\$ 1.7$ billion. ${ }^{15}$ Getting here required strong evidence and the pilot program to prove feasibility and acceptability within the Australian context. Thereafter, modelling and data collection, coupled with comprehensive AIHW reports, demonstrated the program's benefits and showed that expansion would improve its effectiveness. Ironically, the delays in rollout were expected to enable commensurate growth and improvement in efficiency, capacity, and quality of colonoscopy and treatment services, yet NBCSP cases make up less than 10\% of overall colonoscopy demand. ${ }^{16}$ Importantly, increasing participation to $60 \%$ will not just save a further 25000 lives, but also more than $\$ 2$ billion in direct health system costs over the next 25 years, thereby delivering a net cost benefit to government. ${ }^{15}$

The Australian Government Department of Health has overarching responsibility for policy development and program implementation of the NBCSP. This has required cooperation with states and territories, healthcare professionals, cancer experts, primary health providers and the general public, at times with differing and competing objectives. Importantly, there has been outstanding support from the AlHW, with meticulous data analysis and reporting, including comprehensive annual NBCSP monitoring reports. However, the rate of nonmandatory data submissions from colonoscopists and pathologists remains poor ( $<25 \%$ of cases), and linkage with cancer and mortality registries may be a more reliable means of data collection. ${ }^{2,14}$ Also, as yet, only some state cancer registries record pathological staging data, while adenoma histology is not recorded, even though adenoma removal prevents cancer development.

Arguably the most important improvement for the NBCSP would be to increase participation, to save more lives and increase cost-effectiveness. Targeted promotion strategies should be employed for under-participating and disadvantaged groups, including attempts to overcome negative social and cultural stigmas of stool testing. Appropriately, in January 2019, the Australian Government announced $\$ 10$ million in funding for Cancer Council Australia to undertake a national advertising blitz to boost participation.

Colonoscopy access for iFOBT-positive participants is currently suboptimal with a median waiting time of 54 days. $^{2}$ It is recommended that patients have access to colonoscopy as promptly as possible, largely to reduce anxiety induced by a positive test or risk that they will drop out of the program; delays of less than 120 days were not shown to affect cancer staging outcomes. ${ }^{3}$ As the NBCSP demand on overall colonoscopy volume is low, the solutions lie in reducing overuse of low-yield colonoscopy and creating a quarantined pathway for NBCSP cases. Increased participation is also likely to result in less de facto screening with colonoscopy.

The National Cancer Screening Register is being developed as a single database for bowel and cervical screening, to enable recording, analysis and reporting of screening data, with easy access for health professionals and governance bodies. The register can identify screening invitations and responses, and the timing for future invitations. Ideally, a more sophisticated register linking colonoscopy and histology findings will separate out people who should move to a colonoscopic surveillance pathway and no longer be part of iFOBT screening. This might include people with previous advanced adenomas or cancer, and those already in surveillance programs for a strong family history of bowel cancer, hereditary cancer syndromes or inflammatory bowel disease. Another challenge is how best to introduce clinical factors such as limited life expectancy into selection for iFOBT-based screening. GPs are currently best-placed to provide such guidance. 


\section{Can the program fulfil its potential?}

From inception, the NBCSP has been continuously reviewed to refine performance indicators and to consider alternative screening pathways for disadvantaged groups. Policy, governance and quality frameworks have been put in place to ensure appropriate operating standards ${ }^{16,17}$, including the Australian Government using the NBCSP as a lever to ensure high national standards for provision and performance of all colonoscopy. ${ }^{18,19}$ The AlHW reports are used to guide ongoing program development.

Part of this ongoing review includes being responsive to advances and changes. A recent cost-effectiveness analysis demonstrated that using iFOBT remains more cost-effective than screening using plasma and faecal DNA tests, flexible sigmoidoscopy, colonoscopy and CT colonography. ${ }^{20}$ However, new technological and demographic developments may change the balance in the future. Technology review is important as iFOBT appears to be insensitive for detecting serrated polyps, which may be a cancer precursor in $15 \%$ of cases. ${ }^{21}$

Furthermore, lowering the starting age for screening to 45 years would substantially increase the incremental cost-effective ratio by 5-6 fold, from $\$ 3000-\$ 6000$ per life year saved for screening 50-74-year-olds, to $\$ 17000$ \$30 000 per life year saved for 45-74-year-olds. ${ }^{22}$ While cost-effectiveness of screening 45-49-year-olds is currently relatively low, this may change in the future with a substantial rise in bowel cancer incidence in people under 50 years of age. ${ }^{23}$

\section{Conclusion}

Almost 30 years after the emergence of strong evidence proving that FOBT screening saves lives, the NBCSP is finally on the verge of being fully implemented. Crucially, evidence formed the basis for the program's initiation and development, and ongoing evidence will be necessary for its continuation and future improvements. Ultimately, if policy makers, health professionals and the community can work together to get participation to $60 \%$, the program will have a major impact in Australia, potentially saving 84000 lives by 2040 .

\section{Peer review and provenance}

Externally peer reviewed, commissioned.

\section{Competing interests}

$\mathrm{HE}$ is a member of the National Cancer Screening Registry Quality Committee and a paid advisor to the Department of Health WA on bowel cancer screening. JStJ is a member of the Clinical Advisory Group to the National Bowel Cancer Screening Program.

\section{Author contributions}

HE contributed to the structure, writing and revision of the manuscript. JStJ contributed to the structure, writing and revision of the manuscript.

\section{References}

1. Australian Institute of Health and Welfare. Cancer in Australia 2019. Canberra: AlHW; 2019 [cited 2019 Jan 15]. Available from: www.aihw.gov.au/getmedia/8c9fcf520055-41a0-96d9-f81b0feb98cf/aihw-can-123.pdf. aspx?inline $=$ true

2. Australian Institute of Health and Welfare. National Bowel Cancer Screening Program: monitoring report 2018. Canberra: AlHW; 2018 [cited 2019 Feb 11]. Available from: www.aihw.gov.au/getmedia/df120b1a-1bda-49c18611-17a7256e61d0/aihw-can-12.pdf.aspx?inline=true

3. Cancer Council Australia Colorectal Cancer Guidelines Working Party. Clinical practice guidelines for the prevention, early detection and management of colorectal cancer. Sydney: Cancer Council Australia. 2017 [cited 2019 Jan 20]. Available from: wiki.cancer.org.au/australia/ Guidelines:Colorectal_cancer

4. Towler B, Irwig L, Glasziou P, Kewenter J, Weller D, Silagy C. A systematic review of the effects of screening for colorectal cancer using the faecal occult blood test, hemoccult. BMJ. 1998;317(7158):559-65.

5. National Health and Medical Research Council. Guidelines for the prevention, early detection and management of Colorectal Cancer (CRC). Commonwealth of Australia, 1999. [cited 2019 Feb 11]. Available from: webarchive.nla.gov.au/ gov/20170820052135/https://www.nhmrc.gov.au/ guidelines-publications/cp62

6. Australian Government Department of Health and Ageing. The Australian Bowel Cancer Screening Pilot Program and beyond: final evaluation report. Canberra: Commonwealth of Australia; 2005 [cited 2009 Feb 11]. Available from: www.cancerscreening.gov.au/internet/ screening/publishing.nsf/content/9C0493AFEB3FD33CC A257D720005C9F2/\$File/final-eval.pdf

7. Ananda SS, McLaughlin SJ, Chen F, Hayes IP, Hunter AA, Skinner IJ, et al. Initial impact of Australia's National Bowel Cancer Screening Program. Med J Aust. 2009;191(7):378-81.

8. Flitcroft KL, Salkeld GP, Gillespie JA, Trevena LJ, Irwig LM. Fifteen years of bowel cancer screening policy in Australia: putting evidence into practice? Med J Aust. 2010;193(1):37-42.

9. Pignone MP, Flitcroft KL, Howard K, Trevena LJ, Salkeld GP, St John DJ. Costs and cost-effectiveness of full implementation of a biennial faecal occult blood test screening program for bowel cancer in Australia. Med $\mathrm{J}$ Aust. 2011;194(4):180-5. 
10. Cole SR, Tucker GR, Osborne JM, Byrne SE, Bampton PA, Fraser RJ, et al. Shift to earlier stage at diagnosis as a consequence of the National Bowel Cancer Screening Program. Med J Aust. 2013;198(6):327-30.

11. Cenin DR, St John DJ, Ledger MJ, Slevin T, LansdorpVogelaar I. Optimising the expansion of the National Bowel Cancer Screening Program. Med J Aust. 2014;201(8):456-61.

12. Australian Institute of Health and Welfare, Australian Government Department of Health. Analysis of colorectal cancer outcomes for the Australian National Bowel Cancer Screening Program. Asia Pac J Clin Oncol. 2016;12(1):22-32.

13. St John J, Grogan P. Compelling new data on the effectiveness of Australia's National Bowel Cancer Screening Program: a model for best practice? Asia Pac J Clin Oncol. 2016;12(1):7-9.

14. Australian Institute of Health and Welfare. Analysis of bowel cancer outcomes for the National Bowel Cancer Screening Program: 2018. Canberra: AlHW; 2018 [cited 2019 Feb 11]. Available from: www.aihw.gov.au/ getmedia/7878d65f-d1dc-4be0-9b1c-4f897873f56c/aihwcan-113.pdf.aspx?inline $=$ true

15. Lew JB, St John DJB, Xu XM, Greuter MJE, Caruana M, Cenin DR, et al. Long-term evaluation of benefits, harms, and cost-effectiveness of the National Bowel Cancer Screening Program in Australia: a modelling study. Lancet Public Health. 2017;2(7):e331-40.

16. Australian Government Department of Health. National Bowel Cancer Screening Program: policy framework phase four (2015-2020). Canberra: Australian Government Department of Health; 2017 [cited 2019 Feb 11]. Available from: www.cancerscreening.gov. au/internet/screening/publishing.nsf/Content/CDD8E 9C8B95B9C94CA25806A007B6046/\$File/Policy\%20 Framework\%2014112017_KB.pdf
17. Australian Government Department of Health. National Bowel Cancer Screening Program Quality Framework October 2016. Canberra: Australian Government Department of Health; 2016 [cited 2019 Feb 11]. Available from: www.cancerscreening.gov.au/internet/ screening/publishing.nsf/Content/CDD8E9C8B95B9C9 4CA25806A007B6046/\$File/20170329\%20NBCSP\%20 Quality\%20Framework\%20V1.0\%20Endorsed\%20by\%20 SCoS\%20and\%20Published\%20Finalised.pdf

18. Australian Government Department of Health and Ageing. The National Bowel Cancer Screening Program Quality Working Group. Improving colonoscopy services in Australia. Canberra: Australian Government Department of Health and Ageing; 2009 [cited 2019 Feb 11]. Available from: webarchive.nla.gov.au/ gov/20140211194136/http://www.cancerscreening.gov. au/internet/screening/publishing.nsf/Content/nbcs-impcol-ser-0709-cnt

19. Australian Commission on Safety and Quality in Health Care. Colonoscopy clinical care standard. Sydney: ACSQHC; 2018 [cited 2019 Feb 11]. Available from: www.safetyandquality.gov.au/wp-content/ uploads/2018/09/D18-31374-Colonoscopy-BrochureWEB-version-SEP-2018.pdf

20. Lew JB, St John DJB, Macrae FA, Emery JD, Ee HC, Jenkins MA, et al. Evaluation of the benefits, harms and cost-effectiveness of potential alternatives to iFOBT testing for colorectal cancer screening in Australia. Int $J$ Cancer. 2018;143(2):269-82.

21. Zorzi M, Senore C, Da Re F, Barca A, Bonelli LA, Cannizzaro $\mathrm{R}$, et al. Detection rate and predictive factors of sessile serrated polyps in an organised colorectal cancer screening programme with immunochemical faecal occult blood test: the EQuIPE study (Evaluating Quality Indicators of the Performance of Endoscopy). Gut. 2017;66(7):1233-40.

22. Lew JB, St John DJB, Macrae FA, Emery JD, Ee HC, Jenkins MA, et al. Benefits, harms, and cost-effectiveness of potential age extensions to the National Bowel Cancer Screening Program in Australia. Cancer Epidemiol Biomarkers Prev. 2018;27(12):1450-61.

23. Feletto E, Yu XQ, Lew JB, St John DJB, Jenkins MA, Macrae FA, et al. Trends in colon and rectal cancer incidence in Australia from 1982 to 2014: analysis of data on over 375,000 cases. Cancer Epidemiol Biomarkers Prev. 2019;28(1):83-90

\section{Copyright: (C) (i) (2) (2)}

(c) 2019 Ee and St John. This article is licensed under the Creative Commons Attribution-NonCommercial-ShareAlike 4.0 International Licence, which allows others to redistribute, adapt and share this work non-commercially provided they attribute the work and any adapted version of it is distributed under the same Creative Commons licence terms. See: www.creativecommons.org/licenses/by-nc-sa/4.0/ 